\title{
Use of PROMIS and Functional Movement System (FMS) Testing to Evaluate the Effects of Athletic Performance and Injury Prevention Training in Female High School Athletes
}

\author{
Calvin L Cole ${ }^{1,2 *}$, Kostantinos Vasalos ${ }^{1,2}$, Gregg Nicandri ${ }^{1,2}$, Cameron Apt ${ }^{1,2}$, Emmalyn Osterling ${ }^{1,2}$, Zachary Ferrara ${ }^{1,2}$ \\ Michael D Maloney ${ }^{1,2}$, Edward M Schwarz ${ }^{1,2}$ and Katherine Rizzone ${ }^{1,2}$ \\ ${ }^{1}$ Center for Musculoskeletal Research, University of Rochester Medical Center, USA \\ ${ }^{2}$ Department of Orthopedics and Rehabilitation, University of Rochester Medical Center, USA \\ *Corresponding author: Calvin L Cole, Center for Musculoskeletal Research University of Rochester Medical Center 601 Elmwood \\ Avenue, Box 665, Rochester, NY 14642, New York, USA
}

\begin{abstract}
Two major health concerns with female high school athletes are: 1) psychosocial wellness, and 2) sports-related injuries. It is also known that these health concerns are much greater for minority students who attend high school in economically depressed cities. While it has been well-established that exercise is an effective intervention for these health concerns, there are no established outcome measures to quantitatively assess athletic performance and injury prevention training interventions in this population. Previously, we have demonstrated the utility of Patient- Reported Outcomes Measurement Information System (PROMIS) as a robust outcome measure following ACL reconstruction. Functional movement screening (FMS) has been used as a tool to determine injury risk in female collegiate athletes. Since these tools are broadly available, we completed a pilot study of urban underrepresented minority and suburban female high school athletes, to assess the feasibility and utility of these tools to measure changes in this population during 10-weeks of athletic training. No adverse events of the training or study were reported.

A Kaplan-Meier assessment of the pilot revealed that there was high student retention throughout the 10 weeks. In addition, we found no difference in weekly attendance between the students that completed the pilot vs. the dropouts (while they were in the program), indicating that the students were highly motivated to attend when possible. While no significant differences were found for fatigue and physical function, the pilot significantly improved anxiety, peer relationships, pain interference, and trended towards significance for depression ( $\mathrm{p}<0.05)$.In terms of physical performance, bench press, combined Pro Agility, and total FMS were all significantly improved $(\mathrm{p}<0.05)$. Surprisingly, there were 10 students $(67 \%)$ in peril of sports- related injury $(\mathrm{FM}<14)$ at the start of the program, and all but $1(90 \%)$ eliminated this serious risk factor. Collectively, these pilot results demonstrate the feasibility of PROMIS and FMS outcomes to assess the efficacy of physical training interventions, in underrepresented minority female high school students, which warrants investigation in a formal prospective study.
\end{abstract}

Keywords: Underrepresented High School Students; Patient-Reported Outcomes Measurement Information System (PROMIS); Functional Movement Screening (FMS)

\section{Introduction}

Two major health concerns with female high school athletes are: 1) psychosocial wellness, and 2) sports-related injuries [1] It is also known that these health concerns are much greater for minority students who attend high school in economically depressed cities [2]. While it has been well-established that exercise is an effective intervention for these health concerns [3] there are no established outcome measures to quantitatively assess athletic performance and injury prevention training interventions in this population.

Patient-Reported Outcomes Measurement Information System (PROMIS) instruments are a collection of short forms containing a fixed number of items from six PROMIS domains (Depressive Symptoms, Anxiety, Mobility, Pain Interference, Fatigue, and Peer Relationships) along with a single item on Pain Intensity. There are three PROMIS profile lengths, PROMIS-25 was used for this research study and it includes 4 items per domain. The profiles are universal rather than disease specific and assess all domains over the past 
seven days except for Physical Function which has no timeframe specified. Previously, we have demonstrated the utility of PROMIS as a high-throughput outcome measure following ACL reconstruction [4]. Functional movement screening (FMS) is a comprehensive exam that assesses quality of fundamental movement patterns to identify a person's muscular imbalances and asymmetries. A fundamental movement pattern is a basic movement utilized to test range of motion, balance, and stability, concurrently [5]. To successfully complete seven fundamental movement patterns each individual must demonstrate muscular strength, flexibility, range of joint motion, coordination, balance, and proprioception. Each person is scored on a scale ranging from zero to 3 on each of the seven movement patterns and 3 is considered normal. Scores from the seven movements are compiled to obtain comprehensive score [5]. FMS has previously been used as a tool to determine injury risk in high school students [6] and female collegiate athletes [7]. We developed and conducted a pilot study of urban underrepresented minority and suburban female high school athletes, to assess the feasibility and utility of these tools to measure changes in this population during 10 -weeks of athletic training.

\section{Methods}

All human subject research with children was performed following informed consent from a legal guardian and assent from the study subjects, on an IRB approved protocol. Female studentathletes from an urban minority high school (Rochester, NY, n=15) and suburban high schools (Webster, NY, $n=15$ ) were recruited into a 10-week athletic training study at the YMCA in Penfield, NY. Selfreported PROMIS data from the subjects were collected via iPad to a secure server at each session, and FMS baseline and final data were collected at the beginning and end of the 10-week study period, respectively. To obtain scores for FMS the female student-athletes were asked to perform seven movement patterns that included; 1) the deep squat which assesses bilateral, symmetrical, and functional mobility of the hips, knees and ankles, 2) the hurdle step which examines the body's stride mechanics during the asymmetrical pattern of a stepping motion, 3) the in-line lunge which assesses hip and trunk mobility and stability, quadriceps flexibility, and ankle and knee stability, 4) shoulder mobility which assesses bilateral shoulder range of motion, scapular mobility, and thoracic spine extension, 5) the active straight leg raise which determines active hamstring and gastroc-soleus flexibility while maintaining a stable pelvis, 6) the trunk stability push-up which examines trunk stability while a symmetrical upper- extremity motion is performed, and 7) the rotary stability test which assesses multiplane trunk stability while the upper and lower extremities are in combined motion. The data acquisition and athletic training was performed by five certified athletic trainers from the University of Rochester Department of Orthopaedics. As a feasibility pilot study, the primary goals were to demonstrate our ability to: 1) recruit and retain the human subjects throughout the 10-week study period, 2) evaluate global health quantified by PROMIS (depression, anxiety, pain, peer relationships and physical function), and 3) assess risk of injury quantified by FMS testing. All performance data was obtained pre- and post-intervention. PROMIS scores were tabulated as t-scores using data taken from the first and last week of the intervention. To determine each individual's maximal effort on the bench, press a weight was selected based on reps achieved during warm-up, each athlete lay in the supine position, un-racked the bar, and bench pressed the pre-selected weight five times.

The weight was either increased based on the athlete's ability to obtain five repetitions. The test was stopped when the athlete was incapable of reaching five repetitions and the highest weight that they were able to achieve five reps without assistance was taken as their maximal effort. To deem a repetition, complete the individual's hips must stay on the bench (i.e. you can't lift the hips or "thrust" the weight up with your lower body), they must lock out the elbows at the top of the lift, and they are allowed to take a breath or pause at the top in between repetitions. Combined pro agility data was obtained by asking each athlete to start the test in a crouched position, in between two cones which are 10-yards apart. The test goes as follows: From the crouched position, the athlete explodes to the right and touches the line with their right hand, they then explode out of this cut and sprints 10 yards, touching the line with their left hand, and then explodes back through the middle cone. After a short break the test is the repeated but starts by going to the left first. Time starts on the athlete's first move out of the crouched position and ends once they cross the middle line the second time. The average was taken from both right and left test. Statistical analyses to assess changes after the 10-weeks of athletic training were performed using paired t-test for pre/post differences and Kaplan-Meier curve were utilized to assess attendance.

\section{Results \& Discussion}

Fifteen students completed the 10-week training, and no adverse events of the training or study were reported. A KaplanMeier assessment of the Pilot revealed that there was excellent student retention throughout the 10 weeks (Figure 1). We also tracked the attendance of each student, which showed that there was no difference in weekly attendance between the students that completed the Pilot vs. the dropouts $(\mathrm{p}=0.88)$, indicating that the students were highly motivated to attend during the time that they were in the program. The PROMIS data are presented in mean \pm std (Figure 2). While no significant differences were found for fatigue and physical function, the pilot significantly improved anxiety (45.8 \pm 7.4 vs. $41.4 \pm 7.0, \mathrm{p}=0.006$ ), peer relationships ( $51.5 \pm 6.8$ vs.54.1 $\pm 6.5, p=0.02)$, pain interference ( $47.0 \pm 6.4$ vs. $44.1 \pm 7.8, p=0.02)$, and trended towards significance for depression ( $47.0 \pm 7.9$ vs.44.1 $\pm 6.8, p=0.08$ ). This is particularly encouraging since we were not expecting to observe these dramatic effects of the Pilot on such a challenging demographic with a very small cohort of students. The physical performance data are presented in mean \pm std (Figure $3)$. Note that the Pilot significantly improved Bench Press (70.2 \pm 10.7lbs vs. $82.0 \pm 13.9 \mathrm{lbs}, \mathrm{p}=0.0009$ ), Combined Pro Agility (5.8 $\pm 0.5 \mathrm{sec}$ vs. $5.4 \pm 0.4 \mathrm{sec}, \mathrm{p}=0.005)$, and Total FMS (20.3 \pm 4.6 vs. $30.4 \pm 4.5, \mathrm{p}<0.0001)$. Although the improvements in physical performance were predicted from the known effects of athletic training, surprisingly, there were 10 students $(67 \%)$ in peril of sports-related injury $(\mathrm{FMS}<14)$ at the start of the program, and all but $1(90 \%)$ eliminated this serious risk factor. 
A

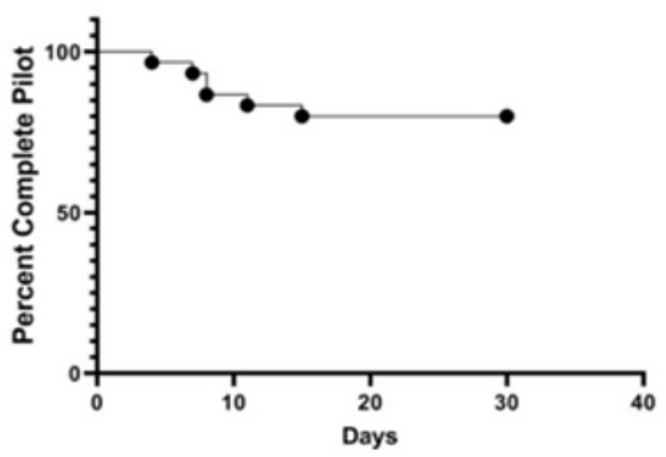

B

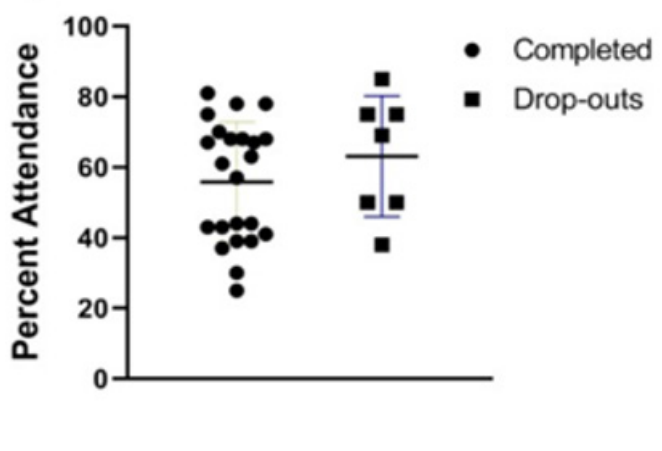

Figure 1: Excellent Retention and Regular Attendance of Students in the CHAMPP Pilot.

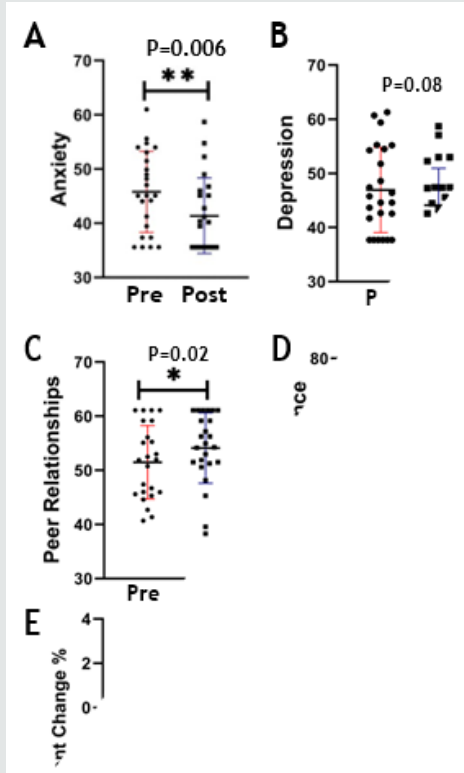

Figure 2: Effects of the CHAMPP Pilot on Students' Anxiety, Depression, Peer Relationships and Pain by PROMIS.

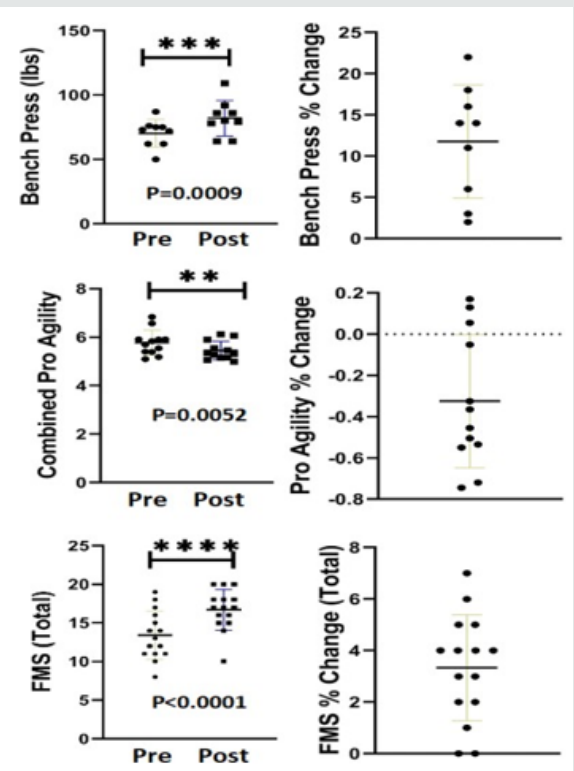

Figure 3: Effects of the CHAMPP Pilot on Students' Athletic Performance and Risk of Injury. 
We enrolled 30 female high school students into the CHAMPP Pilot, and their (A) retention is piloted over the 10 weeks. Note that there were seven students who dropped out within 30 days, and the rest completed the program. To assess compliance, we quantified the (B) percentage of sessions that each student attended normalized to their drop-out or completion date. The percent attendance for each of the seven students that dropped out and the 23 students that completed the Pilot are graphed separately with the mean for each group. Circle represent data collected at baseline. Squares represent data collected after the intervention. PROMIS scores at baseline and after 10 weeks of the Pilot for each student for: (A) Anxiety, (B) Depression, (C) Peer Relationships and (D) Pain Interference. Significant changes from baseline were observed for all except Depression, which almost reached significance $\left({ }^{*} \mathrm{p}<0.05\right)$. Note that negative (E) \% change is the desirable outcome for all measures except Peer Relationships. Circle represent data collected at baseline. Squares represent data collected after the intervention. Student athletic performance data $(n=15)$ were collected in Ortho Metrics TM at baseline and after 10 weeks of the Pilot for each student with the mean and \% change is presented for Bench Press, Combined Pro Agility, and Total FMS. Significant changes from baseline are shown. Note that students with an FMS score below 14 are considered to be at risk for injury, and that the $25 \%$ increase in FMS score for the group was highly significant. Circle represent data collected at baseline. Squares represent data collected after the intervention.

\section{Conclusion}

This Pilot demonstrates the utility of PROMIS and FMS outcome measures to quantify changes in psychosocial wellness and sportrelated injury risk in high school female athletes following a 10- week training program. These findings also support research that exercise improves mood and overall health. However, due to the small sample size of this study, these findings need to be reproduced in a larger, formal study to establish these standards.

\section{Acknowledgement}

This work was supported by research grants from the Konar Foundation and the National Institutes of Health (P30 AR069655).

\section{References}

1. Sabato TM, Walch TJ, Caine DJ (2016) The elite young athlete: strategies to ensure physical and emotional health. Open Access J Sports Med 7: 99-113.

2. Lipson SK, Kern A, Eisenberg D, Breland Noble AM (2018) Mental Health Disparities Among College Students of Color. Journal of Adolescent Health 63(3): 348-356.

3. Heath GW, Parra DC, Sarmiento OL, Andersen LB, Owen N, et al. (2012) Evidence-based intervention in physical activity: lessons from around the world. Lancet 380: 272-281.

4. Papuga MO, Beck CA, Kates SL, Schwarz EM, Maloney MD (2014) Validation of GAITRite and PROMIS as high-throughput physical function outcome measures following ACL reconstruction. Journal of Orthopaedic Research 32: 793-801.

5. Kiesel K, Plisky PJ, Voight ML (2007) Can Serious Injury in Professional Football be Predicted by a Preseason Functional Movement Screen? North American journal of sports physical therapy NAJSPT 2: 147-158.

6. Bardenett SM, Micca JJ, DeNoyelles JT, Miller SD, Jenk DT, et al. (2015) Functional Movement Screen Normative Values and Validity in High School Athletes: Can the Fms Be Used as a Predictor of Injury? International Journal of Sports Physical Therapy 10: 303- 308.

7. Chorba RS, Chorba DJ, Bouillon LE, Overmyer CA, Landis JA (2010) Use of a functional movement screening tool to determine injury risk in female collegiate athletes. North American Journal of Sports Physical Therapy $5: 47-54$.

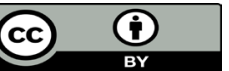

This work is licensed under Creative Commons Attribution 4.0 License

To Submit Your Article Click Here:

Submit Article
DOI: $10.32474 /$ OSMOAJ.2019.03.000160

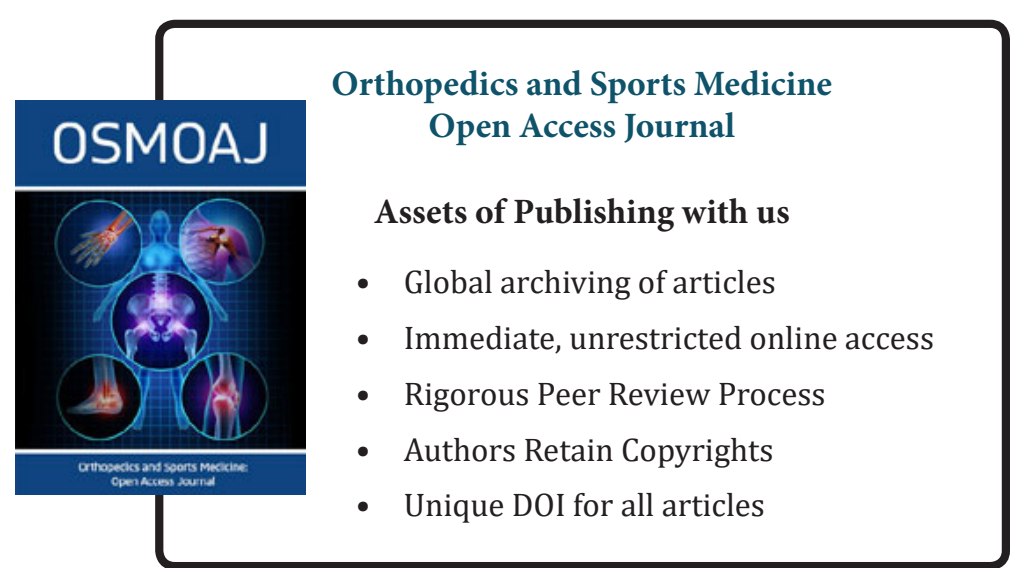

